\title{
Autoeficacia y empatía en docentes mujeres del nivel preescolar
}

Self-efficacy and empathy in female preschool teachers

Recepción del artículo: 15-11-2020 | Aceptación del artículo: 21-12-2020

Angela María Herrera Alvarez

Universidad Norbert Wiener aherrera@uwiener.edu.pe

D https://orcid.org/0000-0002-6399-3850

Maruja Dionisia Baldeón de la Cruz Universidad Norbert Wiener mbaldeon@wiener.edu.pe

https://orcid.org/0000-0003-0851-3938
Para referenciar este artículo:

Herrera, A. M. y Baldeón, M. D. (2020). Autoeficacia y empatía en docentes mujeres del nivel preescolar. Revista ConCiencia EPG, 5(2), 60-69. https://doi.org/10.32654/CONCIENCIAEP

G.5-2.4

\section{Resumen}

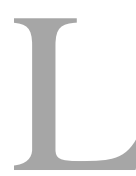

a autoeficacia son las creencias que poseen los docentes sobre sus propias competencias, sin embargo, para aplicarlo de manera adecuado, es necesario contar con otras habilidades más emocionales, como la empatía, solidaridad, compañerismo, entre otras. Bajo esta perspectiva, se desarrolló este estudio, cuyo objetivo fue analizar la relación entre autoeficacia docente y empatía. El estudio tuvo un diseño correlacional y se llevó a cabo en una muestra de 50 docentes mujeres pertenecientes al nivel preescolar de la selva central del Perú, para lo cual, se utilizaron dos instrumentos: Escala de Autoeficacia Docente (Menghi, Oros y Abreu, 2015) y el Test de empatía cognitiva y afectiva (TECA, López- Pérez, Fernández- Pinto y Abad, 2008). Los resultados del estudio revelan que existe una relación positiva muy baja, pero no significativa entre autoeficacia docente y empatía. Con lo cual se concluye que las docentes a pesar de poseer niveles satisfactorios de autoeficacia, requieren poco de la empatía para llegar a sus estudiantes.

Palabras Clave: autoeficacia docente, empatía cognitiva, empatía afectiva. 


\section{Abtract}

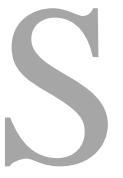

elf-efficacy is a set of beliefs that teachers have about their own competences, however, to apply it properly, it is necessary to have other more emotional skills, such as empathy, solidarity, companionship, among others. From this perspective, this study was developed, the objective of which was to analyze the relationship between teacher self-efficacy and empathy. The study had a correlational design and was carried out in a sample of 50 female teachers belonging to the preschool level of the central jungle of Perú, for which, two instruments were used: Teaching Self-efficacy Scale (Menghi, Oros \& Abreu, 2015 ) and the cognitive and affective empathy test (TECA, López- Pérez, Fernández- Pinto and Abad, 2008). The results of the study reveal that there is a very low, insignificant, positive relationship between teacher self-efficacy and empathy. With which it is concluded that, due to having satisfactory levels of self-efficacy, teachers require little empathy to reach their students.

Key words: teacher self-efficacy, cognitive empathy, affective empathy.

\section{Introducción}

a autoeficacia docente según Yevilao (2019) es la propia opinión que un maestro tiene sobre su capacidad para lograr las metas deseadas en sus estudiantes, y donde su esfuerzo, su dedicación a la enseñanza, las metas que se ha trazado, el afrontamiento del estrés y su nivel de aspiración se pueden ver afectados. Sin embargo, la globalización y la era digital vienen proponiendo cambios en el quehacer de los profesionales docentes, la gran mayoría debe responder a las exigencias que hoy demanda el mundo, es en este sentido que, Flores y Romero (2020) consideran que tanto las personas como profesionales docentes del siglo XXI, son parte de una realidad en la cual se precisa que cuenten y desarrollen capacidades y habilidades que estén alineados con los cambios que viene experimentando la sociedad moderna. La era digital exige que los docentes cambien sus estilos de interactuar unos con otros, así como readecuar sus formas de aprender, enseñar, recibir e intercambiar conocimiento con el propósito de ser cada vez más atractivos y vigentes en una sociedad exigente y competitiva. Sin embargo, en la actualidad el trabajo del docente no solo es el pedagógico, sino el integral, considerando las emociones $\mathrm{y}$ sentimientos de su grupo y comunidad educativa. La docencia implica la gestión de recursos y conocimientos, así como habilidades menos tangibles (Hernández y Ceniceros, 2018). Por ello, García (2018) sostiene que si los docentes desean tener éxito, deben desarrollar conciencia sobre sí mismos, deben conocer con que capacidades cuentan y que competencias requieren para lograr tomar las decisiones acertadas con los cuales puedan lograr sus objetivos de vida y ser eficaces al realizar el trabajo en el cual se desempeñan, por ello es necesario contar con habilidades cognitivas y creencias que les permitan a cada docente ser eficientes. Así mismo Pacheco (2012) sostiene que en estos 
tiempos modernos, "la educación promueve más la necesidad de formar habilidades y competencias, en lugar de transmitir conocimientos e información" (p.20).

Es en ese sentido la importancia de contar con estudios que contribuyan a conocer sobre las prácticas docentes y cómo estas pueden ser desarrolladas de mejor manera cuando el docente analiza su autoeficacia y el desarrollo de su empatía.

\section{Autoeficacia en docentes}

En el año 1946 se funda Corporación RAND (Research and Development), con sedes en Estados Unidos, Países Bajos, Alemania y Reino Unido; Bustamante et al (2017) refieren que esta empresa brindaba servicios que consistía en desarrollar estudios para la mejora de la toma de decisiones en el sector público a través del análisis y de la investigación; en el año 1976 fueron los primeros que realizan una investigación sobre la autoeficacia del docente; su estudio se basó en analizar el rendimiento en lectura de estudiantes de veinte escuelas diferentes; obtuvieron múltiples resultados de los cuales como conclusión llegan que el sentimiento de autoeficacia de los profesores estaba presente y relacionada con otros resultados de estudio y es a partir de ahí que el término autoeficacia docente toma relevancia como tema de investigación en la educación.

La Teoría Cognitivo Social de Bandura se ha aplicado de manera favorable en el campo educativo, Casas y Blanco (2016) consideran que ello se ha dado en tres ámbitos: el análisis de las creencias de autoeficacia de los profesores, la autoeficacia de los estudiantes asociado al rendimiento y la motivación del aprendizaje; y la influencia de los constructos sociocognitivos autorreferentes sobre el desarrollo de la carrera académico-profesional.

Es en ese sentido que Luna (2019) refiere que un docente con fuertes creencias de autoeficacia:

- Utilizará técnicas y metodologías que incentiven la autonomía de los estudiantes de forma que su control sea el mínimo y estos tengan la oportunidad de desarrollar sus competencias y fomentará las condiciones idóneas para el aprendizaje.

- Tomará en cuenta el contexto de su grupo, sus necesidades de aprendizaje y cambiará a las estrategias de enseñanza para con ello asegurar que logren los objetivos establecidos.

- Motivará a sus estudiantes con la finalidad que presenten altos niveles de participación y les brindará reconocimiento por los esfuerzos que realizan.

Para Salvatore et al (2019) los docentes que presentan un bajo nivel de autoeficacia, al ver que sus estudiantes no logran los resultados se rinden con facilidad, la actividad que desarrollan en clase deja de seguir el orden curricular y son de tipo recreativas; para controlar su clase ejercen presión por medio de reglas coercitivas y estrictas y muchos de ellos consideran dedicarse a otras labores y no a la docencia. 


\section{Empatía cognitiva y afectiva en docentes}

Goroshit y Hen (2016) por medio de investigaciones realizadas refieren que los docentes cumplen un rol importante creando entornos y generando un clima óptimo en el aula para que sus estudiantes logren sus objetivos de aprendizaje, ello también significa brindar respuestas a sus necesidades y preocupaciones, por ello la empatía del docente es considerada una habilidad de enseñanza que se requiere si se desea promover un ambiente de aprendizaje positivo, así mismo es la forma que tiene el docente de expresar preocupación y adoptar la perspectiva de sus estudiantes; y en ese sentido los docentes que son empáticos se convierten en ejemplo de moralidad para sus estudiantes, logrando con ello una interacción efectiva, la cual va a mejorar la calidad de la enseñanza y aprendizaje, contribuyendo de esta manera a fomentar un mejor comportamiento motivando el intercambio y la participación.

En esta misma línea Camacho et al (2019), precisan que la empatía en los docentes es relevante en el desarrollo de los estudiantes ya que motiva en estos un mejor desenvolvimiento en sus actividades de aprendizaje, si el docente es empático cultivará en su alumno confianza, seguridad y ello conducirá a que participe y preste mayor atención consolidando de esta manera su conocimiento y construyendo un aprendizaje integral y por ello Putrino et al (2019), consideran que los docentes necesitan contar con un nivel de empatía alto para entender a sus estudiantes, poseer una actitud de diálogo y simpatía, las cuales son parte de las relaciones interpersonales y que intervienen de manera favorable en el proceso educativo; y en este punto es necesario enfatizar lo que indica al respecto Vital et al (2020) "es el de la implicación empática excesiva, que puede dañar la relación interpersonal, el proceso educativo y hasta la propia salud mental del profesional, más propenso a quemarse " (p.5).

El docente de hoy es un facilitador en la clase por esta razón deben tener una formación sólida en inteligencia emocional y optimizar sus habilidades blandas, entre ellos la empatía, con el propósito de sacar lo mejor de sus estudiantes; así mismo autores como Vital et al (2020) mencionan que la empatía es importante en los maestros porque genera un mayor acercamiento, encuentro y aceptación del otro mejorando la relación educativa, promueve en todo momento el desarrollo personal del estudiante, va mejorar el trabajo en equipo o trabajo entre pares $\mathrm{y}$ tendrá influencia en el buen desempeño laboral docente.

\section{Método}

Diseño: el estudio se realizó bajo un diseño no experimental transversal, de alcance correlacional (Hernández, Méndez, Mendoza y Cuevas, 2017), debido a que el objetivo fue analizar la relación entre autoeficacia y empatía en docentes del nivel preescolar de enseñanza.

Participantes: La muestra estuvo constituida por 50 docentes mujeres del sector público, pertenecientes al nivel de educación preescolar de la localidad de Villa Rica en la selva central del Perú cuyas edades van de 22 a 61 años y la media de 38. Así mismo referente al tiempo de 
servicio docente la media es de 8 años y la máxima de 35 . Por otro lado, la profesionalización docente de la muestra seleccionada es heterogénea, se evidenció que el nivel mínimo es de estudiantes universitarias, el máximo de doctorado y la media de licenciadas.

Materiales: Para la recolección de la información se utilizaron la escala de autoeficacia (Menghi, Oros y Abreu, 2015). La escala está compuesta por seis dimensiones: eficacia para influir en la toma de decisiones $(1,2,3,23)$, autoeficacia educativa $(4,5,6,7,8,9,10,11)$, autoeficacia disciplinaria $(12,13)$, eficacia para el logro de la participación parental(14, 15, 16), eficacia para el logro de la participación comunitaria $(17,18,19,20)$ y eficacia para crear un clima positivo en la escuela $(21,22,23,24,25,26)$. Las respuestas a las preguntas del cuestionario son de tipo Likert, que va desde total desacuerdo, algo en desacuerdo, desacuerdo, de acuerdo, muy de acuerdo, así mismo la versión de este cuestionario arrojó un índice de confiabilidad de 0,892 con lo cual representa una elevada confiabilidad. Para evaluar la variable empatía se administró el Test de empatía cognitiva y afectiva - TECA (López- Pérez, Fernández- Pinto \& Abad, 2008) compuesta por cuatro dimensiones: adopción de perspectiva $(1,2,3,4,5,6,7,8)$, comprensión emocional $(9,10,11,12,13,14,15,16,17), \quad$ estrés empático(18,19,20,21,22,23,24,25) y alegría empática $(26,27,28,29,30,31,32$, 33).Las respuestas a las preguntas son de alternativas tipo que van desde la escala de nada, muy poco, algo, bastante y mucho que cuenta con una fiabilidad de 0,782 , es decir representa una aceptable confiabilidad.
Procedimiento: Los instrumentos fueron aplicados en el mes de agosto del año 2019 después de una capacitación pedagógica a docentes en la Ugel Oxapampa perteneciente a la selva central del Perú previa autorización de cada participante. Los cuestionarios fueron entregados con la correspondiente instrucción para ser completados después de la jornada de capacitación, así mismo los datos obtenidos se han tratados con confidencialidad y anonimato de sus respuestas. Y para el análisis de los datos se utilizó el software estadístico SPSS en su versión 24.

\section{Resultados}

La autoeficacia según percepción de las docentes participantes en el estudio es favorable, debido a que los niveles evidenciados van entre regular con un $28 \%$ y alto con un $72 \%$. Así mismo al realizar el análisis entre grupos de edades $y$ profesionalización de las docentes de la muestra seleccionada, se encontró que el grupo de maestras con edades entre 39 y 61 años con grados entre maestría y doctorado presentan un nivel alto de autoeficacia, en comparación al grupo de docentes con edades entre 20 y 38 años que evidencia un $33.3 \%$ en el nivel regular de autoeficacia. Por otro lado la empatía cognitiva afectiva en docentes del nivel preescolar se encuentra en mayor porcentaje en el nivel regular con un 54\% y con una diferencia del nivel alto de $14 \%$, así mismo en el nivel bajo solo se evidencia un 6\%. Así mismo, en cuanto a edad y profesionalización se evidencia que las docentes con edades entre 39 y 61 años evidencian un porcentaje significativo de nivel alto de 
empatía cognitiva afectiva, así mismo en este grupo se encontraron a las docentes con grado de maestría y doctorado, en comparación al grupo de docentes con edades de 22 a 38 años existe una diferencia mínima de $1 \%$ en el nivel alto de empatía cognitiva afectiva.

Tabla 1

Relación con Rho de Espearman entre autoeficacia docente y empatía cognitiva afectiva

\section{Empatía}

$\begin{array}{llll}\text { Rho de } & \text { Autoeficacia } & \text { Coeficiente de } & , 239 \\ \text { Spearman } & \text { docente } & \text { correlación } & , 170\end{array}$

Sig. (bilateral)

50

$\mathrm{N}$

La autoeficacia docente y empatía en la muestra seleccionada presenta una relación muy baja (,239), pero no significativa. Es decir, que los docentes con mayor nivel de autoeficacia, se debería a un mejor desarrollo de la empatía.

Tabla 2

Relación entre las dimensiones de Autoeficacia Docente y dimensiones de empatía

\section{Dimensiones de} Autoeficacia docente

\section{Dimensiones de Empatía}

\begin{tabular}{|c|c|c|c|c|}
\hline Autoeficacia docente & $\begin{array}{l}\text { Adopción de } \\
\text { perspectiva }\end{array}$ & $\begin{array}{l}\text { Comprensión } \\
\text { empática }\end{array}$ & $\begin{array}{l}\text { Estrés } \\
\text { empático }\end{array}$ & $\begin{array}{l}\text { Alegría } \\
\text { empática }\end{array}$ \\
\hline $\begin{array}{l}\text { Eficacia para influir en la } \\
\text { toma de decisiones }\end{array}$ &,- 160 & 035 & 038 &,- 224 \\
\hline Autoeficacia educativa &,- 017 & ,237 & ,246 & 100 \\
\hline Autoeficacia disciplinaria & 157 & ,157 & ,217 & ,162 \\
\hline
\end{tabular}


Eficacia para el logro de la $-, 198$

$-, 088$

,181

,196

participación parental

Eficacia para el logro de la

participación comunitaria

,193

, 002

, $366^{* *}$

, $324^{*}$

Eficacia para crear un clima

$-, 006$

, 027

,138

, 043

positivo en la escuela

Nota: ${ }^{*} \mathrm{p}<.05,{ }^{* *} \mathrm{p}<.01$.

Al realizar el análisis inferencial entre las dimensiones de ambas variables del estudio, se evidenció que en la dimensión eficacia para el logro de la participación comunitaria existe una relación baja directa con estrés empático y alegría empática. Es decir, a mayor eficacia para el logro de la participación comunitaria, mayor es el estrés empático y mayor la alegría empática que desarrollan las docentes mujeres del nivel preescolar.

\section{Discusión}

Los datos del presente estudio revelan que, la autoeficacia docente es satisfactoria, datos que se asemejan a estudios de Fernández y Merino (2012) y, Covarrubias y Mendoza (2015), debido a que las políticas actuales en cuanto a formación del docente están basadas en la autorreflexión, en donde la evaluación del desempeño profesional está regida por procesos que permiten autoevaluar su práctica y con el apoyo del acompañamiento ir mejorándola. Así mismo los datos muestran que el nivel de empatía de los docentes es muy buena, datos que se asemejan con estudio de Putrino, Sasso y Mustaca (2019) y, Vital et al (2020), y ello, se debería a que, el ejercicio docente está relacionado con actitudes positivas que contribuyan a un buen trabajo con los estudiantes.

$Y$ en cuanto a la experiencia docente, los resultados revelan que los docentes con mayor tiempo de servicio presentan promedios menores a los docentes con menos años de experiencia, datos que se asemejan al estudio de Covarrubias y Mendoza (2015), se debería a que las políticas actuales en el sector educación están basados a enfoques muy diferentes a la formación universitaria que tuvieron y esto hace que quizás las docentes con mayor experiencia al tener más información almacenada, les cuesta más tiempo poder reestructurarlo (Punset, 2013).

En cuanto a los resultados del objetivo general, los datos revelan que existe una relación directa muy baja, pero no significativa $(239)$ entre las variables de estudio. Estos resultados están en concordancia con los hallazgos de Abdulah (2019) quien refiere que las personas no 
solo tienen habilidades cognitivas que contribuyen a los procesos de motivación, afecto y acción humana, sino también, cómo motivan y regulan su comportamiento y crean sistemas sociales para organizar y estructurar sus vidas; entonces, la conducta humana es un elemento de un modelo que interactúa para vincularse entre sí con elementos de situaciones ambientales, así como componentes que incluyen afecto/ emoción y cognición individual. En cuanto a los resultados de cada una de las dimensiones de autoeficacia y empatía, solo en la eficacia para el logro de la participación comunitaria se evidenció que tiene una relación baja directa con dos tipos de empatía, la de estrés empático $(, 366)$ y alegría empática (,324) datos que concuerdan con hallazgos de Amirian y Behshad (2016) quienes evidencian que existe una relación significativa en inteligencia emocional $y$ autoeficacia docente, se debería a que inteligencia emocional es un predictor de la autoeficacia docente (Gundlach, Martinko y Douglas, 2003; Amirian y Beshad, 2016). En las dimensiones: eficacia para influir en la toma de decisiones, autoeficacia educativa, autoeficacia disciplinaria, eficacia para el logro de la participación parental, eficacia para crear un clima positivo en la escuela, los datos revelaron que existe una relación muy baja, pero no significativa con la empatía y esto se debería a que el rol del docente en los tiempos actuales busca que sea un agente gestor de sus conocimientos y líder en la promoción social con los agentes comunitarios y cada uno de estas acciones están medidas bajo un instrumento de evaluación que son monitoreadas durante todo el año académico.

\section{Conclusiones}

Por todo lo mencionado se concluye que las docentes a pesar de poseer niveles satisfactorios de autoeficacia, requieren poco de la empatía para llegar a sus estudiantes.

En cuanto a las dimensiones la relación baja, se debería a que las docentes creen tanto en sus capacidades que requieren poco de la empatía para poder llegar a sus estudiantes y comunidad educativa.

\section{Referencias}

Abdullah, S.M. (2019). Social Cognitive Theory: A Bandura Thought Review published in 1982-2012. Journal Psikodimensia, 18 (1), 85-100. http://103.243.177.137/index.php /psi/article/view/1708
Bustamante, E., Bustamante, F., González, G. y Bustamante, L. (2017). Autoeficacia docente de profesores de la Escuela de Bioanálisis de la Universidad de Carabobo. Edumecentro, 9(4), 19-32. http://scielo.sld.cu/scielo.php?scri 
pt=sci arttext\&pid=S207728742017000400003

Camacho, G., Rodas, M. y Carrión, F. (2019). Capacidad de empatía en docentes de Medicina de la Pontificia Universidad Católica del Ecuador. Revista Cubana de Educación Médica Superior, 33(3), 1-17. https://www.medigraphic.com/cgi bin/new/resumen.cgi?IDARTICUL $\underline{0=93201}$

Casas, Y. y Blanco-Blanco, A. (2016). Una revisión de la investigación educativa sobre autoeficacia y teoría cognitivo social en Hispanoamérica. Bordón. Revista de pedagogía, 68(4), 27-47. https://dialnet.unirioja.es/servlet/ articulo?codigo $=5681448$

Covarrubias, C. y Mendoza, M. (2015). Sentimiento de autoeficacia en una muestra de profesores chilenos desde las perspectivas de género y experiencia. Estudios pedagógicos (Valdivia), 41(1), 6378. https://dx.doi.org/10.4067/S0 718-07052015000100004

Fernández, M. y Merino, C. (2012). Resultados psicométricos preliminares de la escala de autoeficacia percibida en maestros de Lima. Psicogente, 15(28). http://revistas.unisimon.edu.co/in dex.php/psicogente/article/view/ $\underline{1878}$

Flores, J. y Romero, J. (2020). Globalización y Educación en Nicaragua, impacto en el sistema educativo $y$ transformación de las culturas escolares. Revista Compromiso Social, 1(3), 45-50. https://revistacompromisosocial.u nan.edu.ni/index.php/Compromiso Social/article/view/64

García, C. (2018). Globalización, internacionalización y educación comparada. EDUCIENCIA, 3(5), 5456.

https://educiencia.uat.edu.mx/ind ex.php/Educiencia/article/view/6 $\underline{8}$

Gonzales, M. (2013). Influencia de la autoeficacia docente colectiva en el profesorado. Psicología Educativa, 19

(1), 3-12. https://doi.org/10.5093/ed2013a $\underline{2}$

Goroshit, M. y Meriav, H. (2016). Teachers' empathy: can it be predicted by selfefficacy? Teachers and Teaching, 22 (7), 805-818. http://dx.doi.org/10.1080/135406 $\underline{02.2016 .1185818}$

Gundlach, M. J., Martinko, M. J., \& Douglas, S. C. (2003). Emotional intelligence, causal reasoning, and the selfefficacy development process. The International Journal of Organizational Analysis, 11(3), 229246.

Hernández, L. y Ceniceros, D. (2018). Autoeficacia docente y desempeño docente, ¿una relación entre variables? Innovación Educativa, 18(78), 171-192. http://www.scielo.org.mx/scielo.p 
hp?script=sci arttext\&pid=S166526732018000300171

Hernándz, R., Méndez, S., Mendoza, C. y Cuevas, A. (2017). Fundamentos de investigación. McGraw Hill

López-Pérez, B., Fernández-Pinto, I., y García, F. A. (2008). TECA: test de empatía cognitiva y afectiva: manual. TEA.

Luna, G. (2019). Autoeficacia docente: estudio de la autoeficacia docente en docentes y estudiantes de último año de Magisterio Infantil [Trabajo de fin de grado. Universidad Zaragoza]. España.

https://core.ac.uk/download/pdf/ 290000114.pdf

Menghi, M., Oros, L.; Abreu, R. (2015). Estudio psicométrico de la Escala de Autoeficacia Docente de Albert Bandura en una muestra argentina. Acta Psiquiatrica y Psicologica de America Latina, 61 (1), 22-32.

Pacheco, G. (2012). Globalización y educación. Red Tercer Milenio. http://190.57.147.202:90/xmlui/b itstream/handle/123456789/728/ Globalizacionyeducacion.pdf?seque $\underline{\text { nce }}=1$

Punset, E. (2013). El sueño de Alicia. La vida y la historia se fundan en la historia más emocionante. Destino

Putrino, N., Sasso, R., \& Mustaca, A. (2018). Sentido del humor y empatía en maestras de primaria: reflexiones sobre su impacto en la enseñanza. Revista ConCiencia EPG, 3(1), 57-71. https://doi.org/10.32654/CONCIE NCIAEPG.3-1.4

Salvatore, E.; Liana, A., y Rosanna, A. (2019). Lifelong learning y autoeficacia del profesorado. International Journal of Developmental and Educational Psychology 1(2), 245-254. http://dehesa.unex.es/bitstream/h andle/10662/10612/02149877 2 01912 245.pdf?sequence=1\&isAll owed $=y$

Tschannen-Moran, M. \& Johnson, D. (2011). Exploring Literacy Teachers' Selfefficacy Beliefs: Potential Sources at Play. Teaching and Teacher Education, 27 (4), 751-761.

Vital, L.; Martínez-Otero, V. y Gaeta, M. (2020). La empatía docente en educación preescolar: un estudio con educadores mexicanos. Educação e Pesquisa, 46. https://www.scielo.br/scielo.php? pid=S151797022020000100546\&s cript $=$ sci arttext\&tlng=es

Yevilao, A. (2019). Autoeficacia: un acercamiento al estado de la investigación en Latinoamérica. Revista Reflexión e Investigación Educacional, 2(2), 91-102. http://revistas.ubiobio.cl/index.ph $\mathrm{p} /$ REINED/article/view/4124/382 2. 